\title{
APPLYING GATA3 IN DIFFERENTIATING UROTHELIAL CARCINOMA FROM PROSTATIC ADENOCARCINOMA: AN IMMUNOHISTOCHEMICAL STUDY
}

\author{
WEAM HAMZAH ABDULLAH ${ }^{1}$, HADEEL ABDULELAH KERBEL ${ }^{2 *}$, RAFID FAKHIR AL HUSSEINI ${ }^{3}$
}

${ }^{1}$ Babl Health Directorate, Al- Hilla teaching Hospital, Hillah, Iraq. ${ }^{2}$ Department of Pathology, Hamourabi College of Medicine/University of Babylon, Iraq. ${ }^{3}$ Department of Surgery, College of Medicine/University of Babylon, Iraq. Email: Hadeelkerbel1974@gmail.com

Received: 02 July 2018, Revised and Accepted: 02 August 2018

\begin{abstract}
Objectives: Urothelial carcinoma and prostatic adenocarcinoma are the most common tumors of genitourinary system. Both tumors can demonstrate a broad morphology or present as poorly differentiated carcinoma occurring in urinary bladder or prostate or both organs that raise the suspicion of a locally extending or metastatic carcinoma from either organs. Accurate distinction between these tumors is mandatory because of different tumor biology and therapeutic protocols. In equivocal tumor morphology, the primary option is to use immunohistochemical panel in surgical pathology that includes differentiation markers that will assist pathologists to avoid misdiagnosis. The aim of this study is immunohistochemical evaluation of GATA3 in urothelial carcinoma and prostatic adenocarcinoma with correlation to different clinicopathological parameters.
\end{abstract}

Methods: We used formalin-fixed paraffin-embedded tissue blocks from 51 patients of urothelial carcinoma and 15 patients of prostatic adenocarcinoma including different grades, stages, and types. Monoclonal antibody for GATA3 was used for immunohistochemical staining of tissue sections, and GATA3 expression was semi-quantitatively scored using H-score method.

Results: Of 51 urothelial carcinomas, 96\% were GATA3 positive with a mean H-score $=212$. No correlation between GATA3 expression and clinicopathological parameters includes grade and stage. Lower GATA3 expression was noted in urothelial carcinoma variants. All of prostatic adenocarcinoma cases did not show GATA3 reactivity.

Conclusion: GATA3 reactivity is a reliable factor to confirm diagnosis of urothelial carcinoma and exclude prostatic adenocarcinoma. The routine use of GATA3 as differentiation marker for urothelial carcinoma may be advocated based on the results of this study.

Keywords: GATA 3, Urothelial carcinoma, Prostatic adenocarcinoma, Immunohistochemical expression.

(C) 2018 The Authors. Published by Innovare Academic Sciences Pvt Ltd. This is an open access article under the CC BY license (http://creativecommons. org/licenses/by/4. 0/) DOI: http://dx.doi.org/10.22159/ajpcr.2018.v11i12.28232

\section{INTRODUCTION}

Bladder cancer is the $9^{\text {th }}$ most common cancer worldwide. More than $90 \%$ are urothelial carcinoma. Urothelial carcinoma is 3-4 times more common in males than females [1]. And its more common after 60 years of age [1]. Tobacco smoking, chemical exposure, and schistosomiasis are major risk factors [2-5]

Urothelial carcinomas morphology ranges from papillary to flat, noninvasive to invasive, and low grade to high grade. It is believed that the accumulation of successive genetic alterations rather than a single genetic event determines a tumor's phenotype and, subsequently, the patient's clinical outcome.

Papillary urothelial carcinoma is divided into low-grade and high-grade categories [6]. It represents 70-80\% of newly diagnosed bladder cancer patients and presents with noninvasive or early invasive disease (stages Tis, Ta, or T1). The prognosis of these patients depends on tumor grade. Whereas all infiltrating carcinomas are considered as high grade, their outcomes depend on the stage of the tumor rather than the grade [7]. The outcome of invasive tumors depends on the stage.

Prostate carcinoma is the second most common cancer in the world [1]. Its incidence increases dramatically with age; more than $75 \%$ of patients are 65 years or older ages. Environmental and genetic factors have important roles. Well-documented familial association with 5-10 times increased risk in men with multiple affected $1^{\text {st }}$ degree relatives [8]. High androgen levels may cause and accelerate the prostate cancer development [9]. Majority of prostatic adenocarcinoma cases are asymptomatic diagnosed during PSA screening [10].
Most prostatic adenocarcinomas are composed of acini arranged in one or more patterns. The diagnosis relies on a combination of architectural and cytological findings. The grade is one of the strongest predictors of biologic behavior in prostate cancer. The Gleason score is recommended for routine use in grading [11].

GATA3 is a transcription factor, encoded by GATA3 gene located on chromosome 10p14 in humans [12] that regulates development and function of various normal tissues and may be expressed in these tissues and in their neoplasms. GATA3 was first identified as an important regulator of T-cell development $[13,14]$. In bladder, GATA3 expression in bladder cancer lower than normal urothelium, this suggested that decrease GATA3 expression may be necessary for tumor initiation or maintenance [15].

\section{METHODS}

This retrospective study was carried out in Babylon training center for Pathology, Faculty of Medicine, Babylon University, during the period from December 2017 to 2018.

Ethical clearance for this study was obtained from the Scientific Committee, College of Medicine, Babylon University.

The study group composed of formalin-fixed paraffin-embedded tissue blocks from 66 cases that were collected from laboratory of histopathology in Al-Hilla Teaching Hospital in Babylon and from some private laboratories in this governorate. 51 cases of urothelial carcinoma of the urinary bladder including 40 males and 11 females with a mean age of 70.2 years, and 15 cases of prostatic adenocarcinoma with a 
mean age of 72.3 years were included in this study. Biopsy type for urothelial carcinoma cases was TURBT, whereas most of the prostatic adenocarcinoma were true-cut biopsy $(n=8)$, TURP $(n=4)$, and open prostatectomy $(n=3)$

From each tissue block, two sections were stained with hematoxylin/ eosin (H and E) staining method and immunohistochemical polydetector plus horseradish peroxidase staining method using monoclonal mouse antihuman GATA3 protein, ready-to-use, Bio SB. Clone L50-823, USA.

The criterion for positive immunohistochemical reaction is dark brown precipitate in the nucleus for GATA3. The score was assessed by calculation of $\mathrm{H}$-score for each slide according to the following equation [16]: $\mathrm{H}$-score $=[1 \times(\%$ cells $1+)+2 \times(\%$ cells $2+)+3 \times(\%$ cells $3+)]$

Where, $3+=$ strong, $2+=$ moderate, and $1+=$ weak staining intensity

Statistical analysis was performed using SPSS version software 24 . $\mathrm{p}<0.05$ was considered statistically significant.

\section{RESULTS}

A total of 51 cases of urothelial carcinoma and 15 cases of prostatic adenocarcinoma were studied, distributed as shown in Table 1.

Immunohistochemical positive results for GATA3 were determined as nuclear staining of malignant cells, and accordingly, we categorized the results using $\mathrm{H}$-score which is semi-quantitative assessment involves intensity of nuclear staining and percentage of tumor cells stained.

Accordingly, H-score ranged from 0 to 300 . As in previous studies [16], in this study, the results were recorded as positive when H-score was more than 10. GATA3 sensitivity in urothelial carcinomas at that score

Table 1: Distribution of urothelial carcinoma and prostatic adenocarcinoma cases according to different clinicopathological parameters (age, gender, tumor grade, and stage)

\begin{tabular}{|c|c|c|}
\hline Diagnosis & $\begin{array}{l}\text { Clinicopathological } \\
\text { parameters }\end{array}$ & n (\%) \\
\hline & Age (years) & \\
\hline \multirow[t]{23}{*}{ Urothelial carcinoma } & $<19$ & $1(1.9)$ \\
\hline & $20-39$ & $0(0)$ \\
\hline & $40-59$ & $5(9.8)$ \\
\hline & $60-79$ & $39(76.5)$ \\
\hline & More than 80 & $6(11.8)$ \\
\hline & Gender & \\
\hline & Male & $40(78.4)$ \\
\hline & Female & $11(21.6)$ \\
\hline & Grade & \\
\hline & Low & $31(60.8)$ \\
\hline & High & $20(39.2)$ \\
\hline & Stage & \\
\hline & $\mathrm{Ta}$ & $3(5.8)$ \\
\hline & T1 & $26(50.9)$ \\
\hline & $\mathrm{T} 2$ & $18(35.3)$ \\
\hline & T3 & $2(3.9)$ \\
\hline & $\mathrm{T} 4$ & $2(3.9)$ \\
\hline & Microscopic type & \\
\hline & Papillary & $41(80.3)$ \\
\hline & Conventional infiltrating & $6(11.7)$ \\
\hline & Infiltrating with & $4(7.8)$ \\
\hline & divergent differentiation & \\
\hline & Age (years) & \\
\hline \multirow[t]{7}{*}{ Prostatic adenocarcinoma } & $40-59$ & $2(13.3)$ \\
\hline & $60-79$ & $9(60)$ \\
\hline & More than 80 & $4(26.7)$ \\
\hline & Gleason grade & \\
\hline & 6 & $2(13.3)$ \\
\hline & 7 & $6(40)$ \\
\hline & $8-10$ & $7(46.7)$ \\
\hline
\end{tabular}

was $96 \%$. All positive staining results were non-focal and most cases $73 \%$ (37/49) show diffuse moderate to strong staining (Figs. 1 and 2).

No significant correlation of GATA3 immunostaining with grade and stage of tumor ( $p>0.05)$. Extremely significant correlation of GATA3 immunostaining with type of tumor $(\mathrm{p}<0.01)$ as shown in Table 2.

While all of the 15 prostatic adenocarcinoma cases showed completely negative staining, the specificity of GATA3 in urothelial carcinoma was $100 \%$. Our results also revealed a highly significant difference in GATA3 immunoexpression for urothelial carcinoma \& prostatic adenocarcinoma $(\mathrm{p}<0.01)$.

\section{DISCUSSION}

Most of the prostatic adenocarcinoma and urothelial carcinoma cases can be distinguished using the routine $\mathrm{H}$ and $\mathrm{E}$ method, but there are some challenging cases in which the distinction between the two is not possible.

In poorly differentiated carcinoma affecting prostate and bladder without clear-cut morphologic features to ascertain a specific diagnosis, immunohistochemical evaluation is mandatory because it may represent a local extension of tumor from one organ to the other or a case of two primary tumors.

There are several immunohistochemical markers for prostatic adenocarcinoma including PSA, AMACR, P501, and NKX3.1 can be used with high sensitivity and specificity. However, in rare cases, all markers are negative so prostatic origin cannot be excluded from the study.

For urothelial carcinoma, there is no single immunohistochemical marker with constant and high sensitivity and specificity; therefore, urothelial origin could be proved using a panel of markers such as HMWK, p63, S100P, thrombomodulin, and uroplakin.

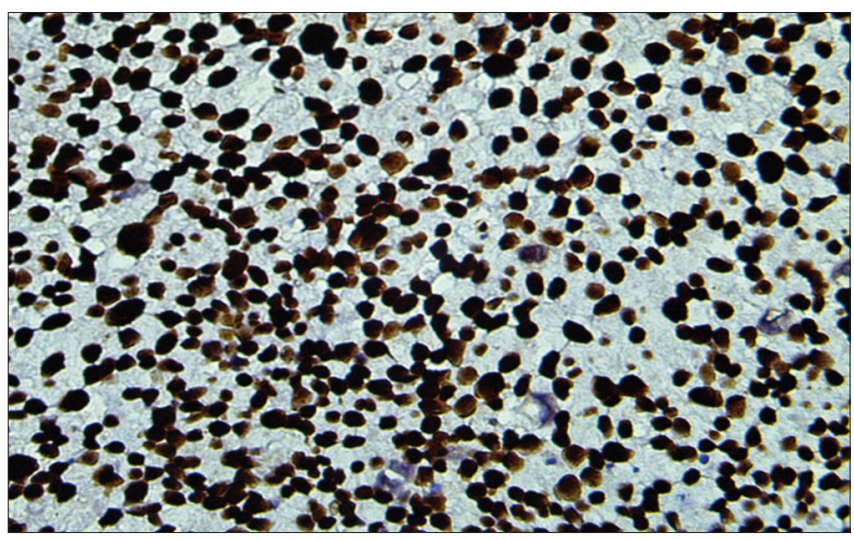

Fig. 1: Infiltrating high grade urothelial carcinoma with high H-score for GATA 3 (X400)

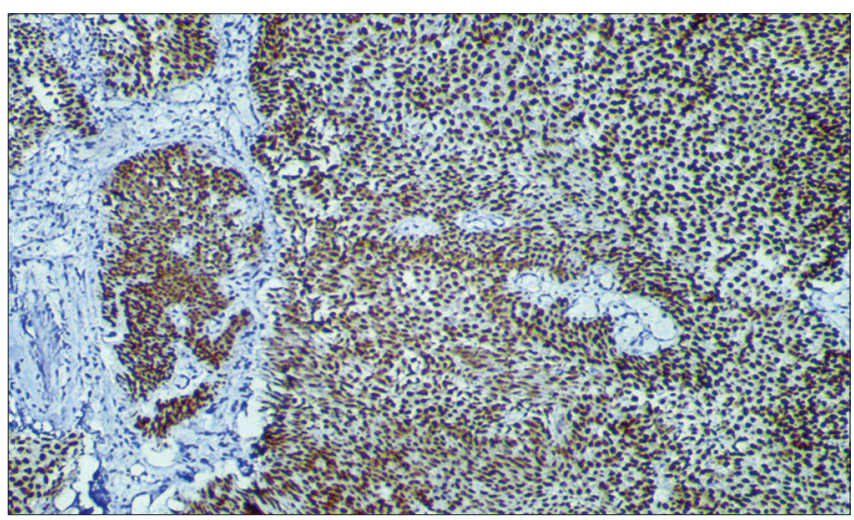

Fig. 2: Low grade Papillary urothelial carcinoma, with high $\mathrm{H}-$ score for GATA 3 ( X200) 
Table 2: Correlation of GATA3 reactivity with urothelial carcinoma grades, stages, and microscopic types

\begin{tabular}{llll}
\hline Clinicopathological parameters & $\mathbf{n}(\mathbf{\%})$ & & Total \\
\cline { 2 - 4 } & Positive & Negative & p value \\
\hline Grade & $31(100)$ & $0(0)$ & $31(60.8)$ \\
Low & $18(90)$ & $2(10)$ & $20(39.2)$ \\
High & $3(100)$ & $0(0)$ & $3(5.8)$ \\
Stage & $26(100)$ & $0(0)$ & $26(50.9)$ \\
Ta & $16(89)$ & $2(11)$ & $18(35.3)$ \\
T1 & $2(100)$ & $0(0)$ & $2(3.9)$ \\
T2 & $2(100)$ & $0(0)$ & $2(3.9)$ \\
T3 & $41(100)$ & $0(0)$ & $41(80.3)$ \\
T4 & $6(100)$ & $0(0)$ & $6(11.7)$ \\
Microscopic type & $2(50)$ & $2(50)$ & $4(7.8)$ \\
Papillary & & & 0.378 \\
Conventional infiltrating & & \\
Infiltrating with divergent differentiation & &
\end{tabular}

This study presents a review of 66 primary tumors including 51 cases of urothelial carcinoma classified according to the WHO classification 2016 [16] and 15 cases of prostatic adenocarcinoma graded according to Gleason grade group system 2015 [10], with the aim to investigate the frequencies of various clinicopathological and histopathological features and their correlations to GATA3 protein expression.

In the current study, we used a semi-quantitative method to evaluate expression level of GATA3 called H-score that incorporated the intensity of nuclear immune reactivity and the proportion of positively stained cell nuclei. We found that mean H-score was 212 (range 30-285).

In reviewing previous studies, most analyzed GATA3 expression using different scoring methods. Oh et al. [17] depended on more than $10 \%$ of cells stained to be positive regardless intensity. Liu et al. [18] considered $<5 \%$ of cells stained to be a negative result. Recently, two studies used $\mathrm{H}$-score method for GATA3 evaluation in urothelial carcinoma. Clark et al. [19] have shown that mean $\mathrm{H}$-score for GATA3 expression in urothelial carcinoma was 170 depending on more than 10 as cutoff value for positive results. Leivo et al. [20] have found that the median $\mathrm{H}$-score was 240 (range 10-300) without specifying the cutoff value in the article. In our work, we used a low $\mathrm{H}$-score $(>10)$ as cutoff value.

Reports of GATA3 sensitivity in urothelial carcinoma extend from $67 \%$ to $99 \%$. In the current study, GATA3 had high sensitivity in urothelial carcinoma among all published studies, where it was $96 \%(49 / 51)$ that was close to Clark et al. [19] study 95\% (21/22), Miettinen et al. [23] study 90\% (49/54), and Leivo et al. [20] study 99\% (88/89), who used the same source of primary antibody (Bio SB, Clone L50823 ) of our study. This differs from GATA3 sensitivity in two larger studies, Higgins et al. [21] found $67 \%$ of 308 cases and Oh et al. [17] found $84.8 \%$ of 138 cases. The difference may be excused due to larger study groups, different primary antibody source, and inherent nature of tissue blocks.

Diffuse strong staining reaction (H-score $>150$ ) was noticed in $73 \%$ of urothelial carcinoma cases. Liu et al. [18] found $65 \%$ of 72 cases and Chang et al. [22] found $89 \%$ of 35 cases revealed strong diffuse staining. This variation in degrees of expression may be resulted from varied scoring methods and different case selection methods, where all cases used in both studies were high-grade muscle invasive.

In keeping with Leivo et al. [20] study, along with our study, has demonstrated that no significant association between GATA3 expression and urothelial carcinoma grade $(p=0.07)$ and stage $(p=0.378)$. On the other hand, Higgins et al. [21] reported that higher GATA3 expression in low grade $(95 \%)$ compared with high-grade $(57 \%)$ urothelial carcinoma. The larger study group used by Higgins may be the cause of different rates.
Significant association was reported between GATA3 expression and microscopic type $(\mathrm{p}<0.01)$ in our study. All conventional urothelial carcinomas (papillary and infiltrating) were GATA3 positive, while four urothelial carcinomas with divergent differentiation showed low to negative GATA3 immunoreactivity, but the number of variant cases included in our study was too small to draw any definitive conclusion in this regard.

Similar observations were found by Miettinen et al. [23] who reported 5/54 GATA3 negative cases including urothelial carcinomas with squamous differentiation. In the same context, Rao et al. [24] study stated that GATA3 expressed in 2/11 (18\%) of urothelial carcinomas with glandular differentiation.

In contrast to our results, Helmy et al. [25] found that $70 \%$ of 10 urothelial carcinoma with squamous differentiation cases were GATA3 positive $(p=0.01)$. The variability in reported results could be attributed to different methodology used including case selection and source of primary antibody.

There were several studies assessing the expression of GATA3 in urothelial carcinoma in comparison to prostatic adenocarcinoma. Highly significant correlation was found $(\mathrm{p}<0.01)$ in the present study, where all studied prostatic adenocarcinoma cases were not express any GATA3 reactivity. In all of the above-mentioned studies [19,20,23,26], none of studied prostatic adenocarcinoma cases showed GATA3 positivity, except in Miettinen et al. study [23] who reported 2\% (2/95) of prostatic adenocarcinoma cases with positive reaction.

\section{CONCLUSION}

The high sensitivity of GATA3 makes itthefirst-lineimmunohistochemical marker for diagnosing urothelial carcinoma, whether as primary tumor in the bladder or metastatic carcinoma from urinary bladder in other sites, along with clinical and morphologic findings. GATA3 seems to be less sensitive in detecting variant urothelial carcinomas in comparison with conventional urothelial carcinoma.

\section{AUTHORS' CONTRIBUTION}

Weam Hamzah Abdullah has majorly performed the experiment in the laboratory, collecting tissues samples, data analysis, and editing the article with references. Hadeel Abdulelah Kerbel has provided the design, intellectual content, innovations, and protocol for conducting the experiment along with membership. Rafid Fakhir Al Husseini has a minor role in conducting the experiment in the laboratory, analysis of obtained data, and sincerely authored article.

\section{CONFLICTS OF INTEREST}

The authors declare that there are no conflicts of interest regarding the publication in this article. 


\section{REFERENCES}

1. Global Burden of Disease Cancer Collaboration, Fitzmaurice C, Allen C, Barber RM, Barregard L, Bhutta ZA, et al. Global, regional, and national cancer incidence, mortality, years of life lost, years lived with disability, and disability-adjusted life-years for 32 cancer groups, 1990 to 2015: A Systematic analysis for the global burden of disease study. JAMA Oncol 2017;3:524-48.

2. Freedman ND, Silverman DT, Hollenbeck AR, Schatzkin A, Abnet CC. Association between smoking and risk of bladder cancer among men and women. JAMA 2011;306:737-45.

3. Reulen RC, Kellen E, Buntinx F, Brinkman M, Zeegers MP. A metaanalysis on the association between bladder cancer and occupation. Scand J Urol Nephrol Suppl 2008;218:64-78.

4. Abol-Enein H. Infection: is it a cause of bladder cancer. Scand J Urol Nephrol Suppl 2008;218:79-84.

5. Minakshi G, Jyoti D, Rakesh KM, Harish D. Therapies in cancer treatment: An overview. Int J Pharm Pharm Sci 2015;7:1-9.

6. Amin MB, Smith SC, Reuter VE, Epstein JI, Grignon DJ, Hansel DE, et al. Update for the practicing pathologist: The international consultation on urologic disease-European association of urology consultation on bladder cancer. Mod Pathol 2015;28:612-30.

7. Amin MB. Histological variants of urothelial carcinoma: Diagnostic, therapeutic and prognostic implications. Modern Pathol 2009;22:S96-118.

8. Zeegers MP, Jellema A, Ostrer H. Empiric risk of prostate carcinoma for relatives of patients with prostate carcinoma: A meta-analysis. Cancer 2003;97:1894-903.

9. Zhu C, Luong R, Zhuo M, Johnson DT, McKenney JK, Cunha GR, et al. Conditional expression of the androgen receptor induces oncogenic transformation of the mouse prostate. J Biol Chem 2011;286:33478-88.

10. Hori S, Blanchet JS, Mcloughlin J. From prostate-specific antigen (PSA) to precursor PSA (proPSA) isoforms: A review of the emerging role of proPSAs in the detection and management of early prostate cancer. BJU Int 2013;112:717-28.

11. Epstein JI, Zelefsky MJ, Sjoberg DD, Nelson JB, Egevad L, MagiGalluzzi $\mathrm{C}$, et al. A contemporary prostate cancer grading system: A Validated alternative to the gleason score. Eur Urol 2016;69:428-35.

12. Yamashita M, Ukai-Tadenuma M, Miyamoto T, Sugaya K, Hosokawa H, Hasegawa A, et al. Essential role of GATA3 for the maintenance of Type 2 helper T (Th2) cytokine production and chromatin remodeling at the Th2 cytokine gene loci. J Biol Chem 2004;279:26983-90.

13. Mohd KS, Hassan MA, Azmin W, Dharmaraj S. A review of potential anticancers from antimicrobial peptides. Int $\mathrm{J}$ Pharm Pharm Sci 2015;7:19-24.

14. McCleskey BC, Penedo TL, Zhang K, Hameed O, Siegal GP, Wei S, et al. GATA3 expression in advanced breast cancer: Prognostic value and organ-specific relapse. Am J Clin Pathol 2015;144:756-63.

15. Li $\mathrm{Y}$, Ishiguro $\mathrm{H}$, Kawahara $\mathrm{T}$, Miyamoto $\mathrm{Y}$, Izumi $\mathrm{K}$, Miyamoto $\mathrm{H}$, et al. GATA3 in the urinary bladder: Suppression of neoplastic transformation and down-regulation by androgens. Am J Cancer Res 2014;4:461-73.

16. Ishibashi H, Suzuki T, Suzuki S, Moriya T, Kaneko C, Takizawa T, et al. Sex steroid hormone receptors in human thymoma. J Clin Endocrinol Metab 2003;88:2309-17.

17. Oh WJ, Chung AM, Kim JS, Han JH, Hong SH, Lee JY, et al. Differential immunohistochemical profiles for distinguishing prostate carcinoma and urothelial carcinoma. J Pathol Transl Med 2016;50:345-54.

18. Liu H, Shi J, Wilkerson ML, Lin F. Immunohistochemical evaluation of GATA3 expression in tumors and normal tissues: A useful immunomarker for breast and urothelial carcinomas. Am J Clin Pathol 2012;138:57-64.

19. Clark BZ, Beriwal S, Dabbs DJ, Bhargava R. Semiquantitative GATA3 immunoreactivity in breast, bladder, gynecologic tract, and other cytokeratin 7-positive carcinomas. Am J Clin Pathol 2014;142:64-71.

20. Leivo MZ, Elson PJ, Tacha DE, Delahunt B, Hansel DE. A combination of p40, GATA-3 and uroplakin II shows utility in the diagnosis and prognosis of muscle-invasive urothelial carcinoma. Pathology 2016;48:543-9.

21. Higgins JP, Kaygusuz G, Wang L, Montgomery K, Mason V, Zhu SX, et al. Placental S100 (S100P) and GATA3: Markers for transitional epithelium and urothelial carcinoma discovered by complementary DNA microarray. Am J Surg Pathol 2007;31:673-80.

22. Chang A, Amin A, Gabrielson E, Illei P, Roden RB, Sharma R, et al. Utility of GATA3 immunohistochemistry in differentiating urothelial carcinoma from prostate adenocarcinoma and squamous cell carcinomas of the uterine cervix, anus, and lung. Am J Surg Pathol 2012;36:1472-6.

23. Miettinen M, McCue PA, Sarlomo-Rikala M, Rys J, Czapiewski P, Wazny K, et al. GATA3: A multispecific but potentially useful marker in surgical pathology: A systematic analysis of 2500 epithelial and nonepithelial tumors. Am J Surg Pathol 2014;38:13-22.

24. Rao Q, Williamson SR, Lopez-Beltran A, Montironi R, Huang W, Eble JN, et al. Distinguishing primary adenocarcinoma of the urinary bladder from secondary involvement by colorectal adenocarcinoma: Extended immunohistochemical profiles emphasizing novel markers. Mod Pathol 2013;26:725-32.

25. Helmy NA, Khalil HK, Kamel NN, AboelFadl DM. Role of GATA3, CK7, CK20 and CK14 in distinguishing urinary bladder squamous cell carcinoma and urothelial carcinoma with squamous differentiation. Egypt J Pathol 2015;35:133-8.

26. Humphrey PA, Moch H, Cubilla AL, Ulbright TM, Reuter VE. The 2016 WHO classification of tumours of the urinary system and male genital organs-part B: Prostate and bladder tumours. Eur Urol 2016;70:106-19.26. 